\title{
Órganos androconiales abdominales peculiares en Theages Walker, 1855 (Lepidoptera: Arctiidae)
}

\author{
Remarkable abdominal androconial organs in Theages Walker, 1855 \\ (Lepidoptera: Arctiidae)
}

\author{
Juan Grados ${ }^{1}$
}

Presentado: $07 / 05 / 2004$

Aceptado: $\quad 21 / 06 / 2004$

\section{Resumen}

Se ha encontrado órganos androconiales en los terga IV, V, VI y VII en especies del género Theages Walker, 1855. Se estudia la morfología de los órganos androconiales y su variación interespecífica.

Palabras clave: Arctiidae, órganos androconiales, Perú, Theages.

\begin{abstract}
Androconial organs on terga IV, V, VI and VII in species of the genus Theages Walker, 1855 are described, including its morphology and interspecific variation.
\end{abstract}

Keywords: Arctiidae, androconial organs, Peru, Theages.

\section{Introducción}

Los órganos sexuales secundarios de machos de Lepidoptera, los cuales se presume ser diseminadores de feromonas, son ampliamente diversificados y están localizados en las patas, tórax y abdomen, variando desde un simple penacho de pelos hasta complejos órganos eversibles, mostrando elaborados métodos de secreción, almacenamiento y liberación (Birch 1972, Birch et al. 1990, Eltringham 1925, Scoble 1992).

Se ha localizado órganos androconiales masculinos de Arctiidae en las alas, patas y en la parte ántero-ventral del abdomen (androconias subabdominales). Estos últimos denominados como «valva ventral» por Hampson (1898), estudiados con algún detalle por Barth (1953), y analizados posteriormente por Weller et al. (2000). Estos últimos han propuesto nuevos nombres para tales órganos: bolsillo simple y bolsillo doble, si intervienen en la formación de tales órganos los sterna II y III, o II, III y IV, respectivamente.

\footnotetext{
${ }^{1}$ Museo de Historia Natural, Universidad Nacional Mayor de San Marcos, Apartado 14-0434, Lima 14, Perú.

E-mail Juan Grados: gradosjuan@hotmail.com
}

Los órganos androconiales ubicados en la parte póstero-ventral del abdomen, entre el sétimo y octavo segmento abdominal (coremata), se conocen desde hace mucho tiempo (Barth 1956, Padgen 1957), y están comprometidos en la liberación de derivados de pirrolizidina (Boppré 1990, Conner et al. 1981). Se ha demostrado que en algunas especies el desarrollo ontogenético de los coremata depende de la cantidad ingerida de alcaloides de pirrolizidina en la etapa larval (Boppré \& Schneider 1989, Davenport \& Conner 2003).

\section{Material y métodos}

Durante colectas de campo con trampa de luz mixta $(250 \mathrm{~V})$ llevadas a cabo en los bosques montanos del departamento de Cuzco (Perú), se observó que especímenes machos de Theages griseatum (Rothschild) (Fig. 1) exhibían los órganos androconiales de la parte dorsal del abdomen, al presionar las pleuras (Fig. 2). Con el objetivo de estudiar la morfología de tales órganos androconiales, se procedió a preparar algunos especímenes en $\mathrm{KOH}$ al $10 \%$ en baño maría por 10 minutos. Se retiró las escamas con pinceles delgados, se cortó la parte lateral del abdomen y se pre- 


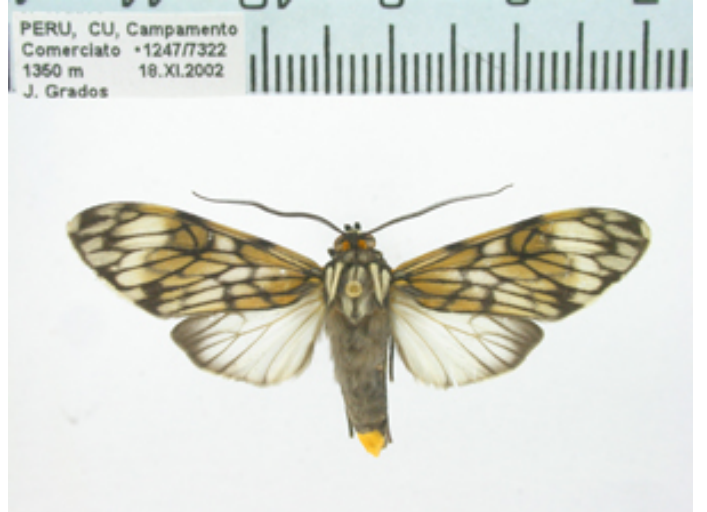

Figura 1 . Theages griseatum Walker, macho

paró láminas con bálsamo de Cánada. Los dibujos se realizaron con cámara lúcida.

\section{Resultados y discusión}

Se conoce que algunas especies de Arctiidae presentan coremata (Padgen 1957, Häuser \& Boppré 1997, Weller et al. 2000,), así como órganos androconiales subabdominales (Barth 1953, Weller et al. 2000). La presencia de órganos androconiales en forma de penacho de pelos en el abdomen ha sido estudiada en algunos otros grupos de lepidópteros (Eltringham 1925, Speidel et al. 1996, Birch et al. 1990, Clearwater 1975), pero se conoce muy poco de su presencia en los Arctiidae neotropicales.

El género Theages fue creado por Walker (1855: 721) al describir la especie Theages leucophaea (Walker, 1855:722) con especímenes provenientes de Brasil. En el catálogo de los Syntomidae (=Ctenuchinae) de Hampson (1898), el género no fue considerado y la especie tipo fue incluida en Eucereon Hübner. Este criterio es seguido posteriormente por otros autores como Zerny (1912) y Draudt (1915-1917). En una nota preliminar, Travassos (1962) reestablece el género Theages Walker, con la inclusión de ocho especies: Theages leucophaea Walker, T. flavicaput (Hampson), T. decorum (Schaus), T. xanthura (Schaus), T. albidius (Rothschild), T. bricenoi (Rothschild), $T$. griseatum (Rothschild) y T. hoffmani Travassos. Travassos (1962) señala que el género es muy característico por ser el único grupo donde los machos presentan glándulas odoríferas situadas dorsalmente en surcos transversales, solamente observables cuando se comprime el abdomen.

Al realizar la preparación y el retiro de las escamas de los terga abdominales de especímenes machos de Theages griseatum (Rothschild), se observa que en las áreas ánterolaterales de los segmentos IV, V, VI y VII, hay un conjunto de cerdas, que se disponen con dirección hacia el margen mesal, en una invaginacion del margen anterior del mismo segmento y que están cubiertos por el segmento abdominal anterior (Fig. 3). La disposición telescópica de los segmentos, hace que

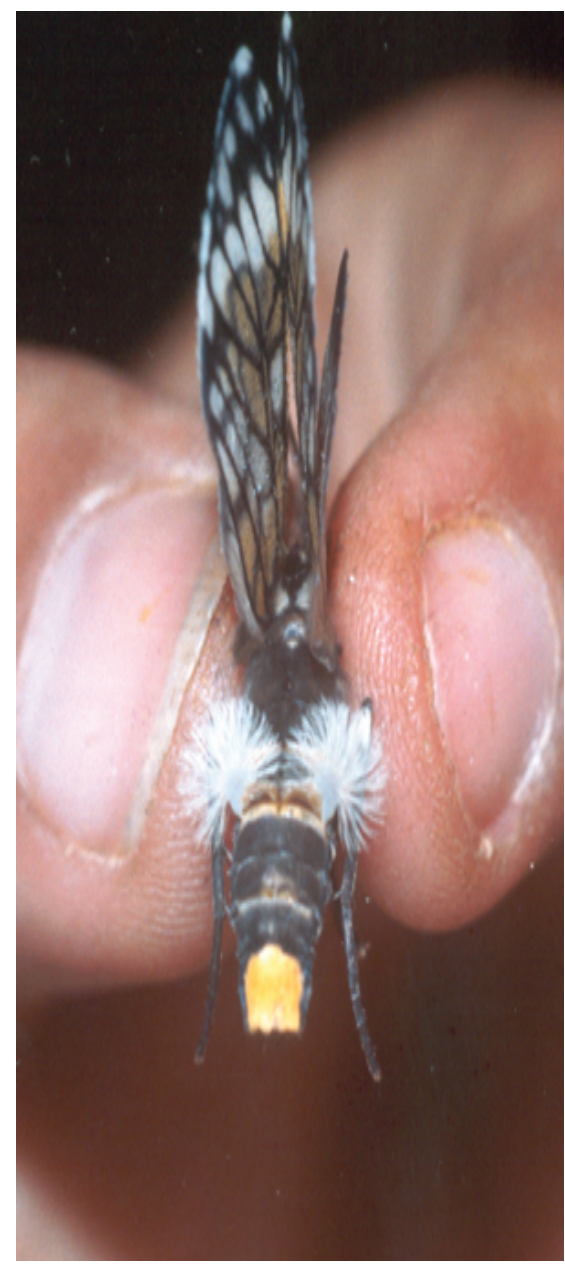

Figura 2 . Penachos de pelos dorsales de Theages griseatum Walker, en espécimen vivo. 


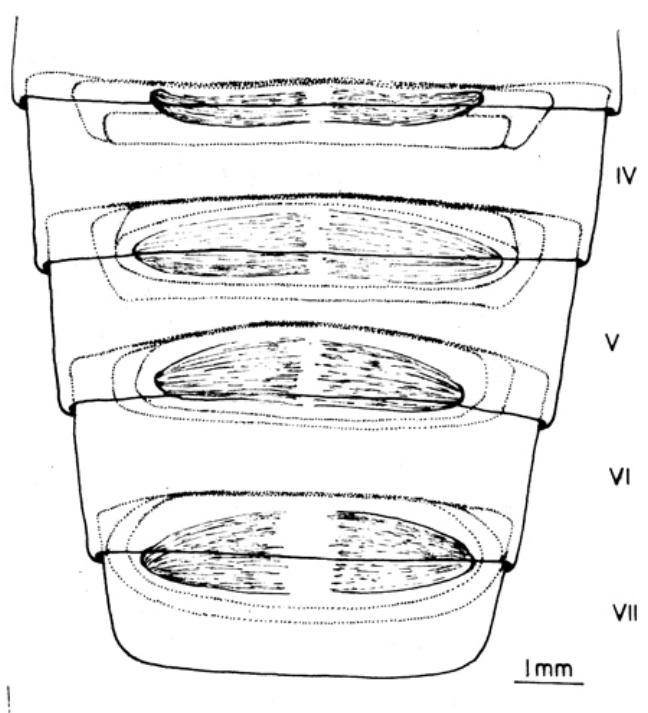

Figura 3 . Penachos de pelos dorsales (órganos androconiales) de Theages griseatum Walker, sobre los terga IV, V, VI y VII del abdomen.

en especímenes vivos no sea fácil poder observar tales órganos androconiales, los cuales denomino como «penachos de pelos dorsales». El margen posterior del III segmento abdominal, se yuxtapone al margen anterior del IV segmento, ocultando el órgano androconial. Lo mismo ocurre con el IV, V y VI segmentos con los inmediatos posteriores correspondientes. Cuando se realiza algo de presión sobre las pleuras, los penachos de pelos son protruidos, tal como se observa en la Figura 2. Órganos androconiales similares han sido reportados en los márgenes anteriores de los sterna IV al VI de Amerila catinca (Hampson), un arctiido del Viejo Mundo (Häuser \& Boppré 1997).

De las ocho especies registradas por Travassos (1962), en la colección del Museo de Historia Natural de la Universidad Nacional Mayor de San Marcos (MUSM) se encuentran cinco: Theages flavicaput, $T$. griseatum, T. xanthura, $T$. decorum y $T$. albidius. Se ha realizado la preparación de dos especímenes de cada especie, a excepción de $T$. albidius, del cual se preparó un solo espécimen, encontrándose variación de los órganos androconiales a nivel interespecífico. $T$. flavicaput (Hampson) presenta el primer par de penachos de pelos dorsales, sobre el IV segmento abdominal, densos y grandes. Los penachos de pelos dorsales sobre el V, VI y VII segmentos son menos desarrollados. El primer par de penachos de pelos dorsales de T. decorum se caracteriza por ser densos $\mathrm{y}$ desarrollados. Los de los otros segmentos van disminuyendo en abundancia y tamaño, siendo los del VI segmento, los más pequeños. Los penachos de pelos del IV segmento de $T$. xanthura son abundantes y desarrollados, mientras que de los otros segmentos son algo más grandes y poseen abundantes escamas oscuras en las invaginaciones donde descansan. Si bien Travassos (1962) menciona que todas las especies poseen pelos dorsales, al hacer la preparación del abdomen de $T$. albidius no he podido encontrar los penachos de pelos dorsales. Lo que se puede observar es la típica invaginación sobre el IV segmento abdominal, pero no penachos de pelos. Lamentablemente se cuenta con pocos especímenes de esta especie para hacer más preparaciones.

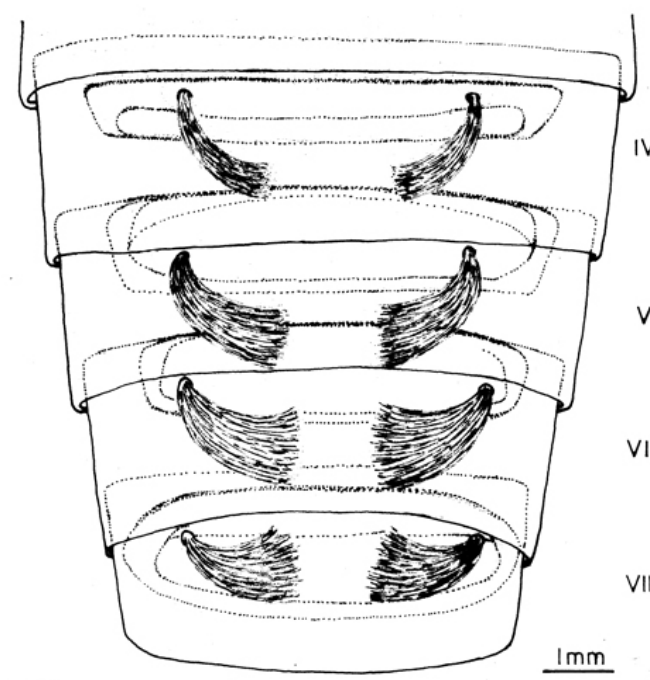

Figura 4 . Penachos de pelos dorsales de Theages griseatum Walker, hipotéticamente evertidos. 
Al parecer, no hay variación intraespecífica en estos órganos androconiales.

No se tiene datos acerca de la función de tales órganos androconiales. La figura 4 muestra la manera hipotética como se protruirían los penachos, tal como también se puede observar en la figura 2. Parte del abdomen es extendido, dejando libre el margen anterior del segmento donde se encuentran los penachos de pelos. Se desconoce si estos penachos están asociados a la liberación de alguna determinada sustancia, aunque tal vez estén comprometidos en el comportamiento del cortejo y apareamiento, por lo cual son necesarios trabajos sobre etología reproductiva.

\section{Agradecimiento}

La foto de T. griseatum (Rothschild) en el campo, mostrando los órganos androconiales, fue tomada por José Roque y del espécimen seco y montado por Mathieu Joron. Sugerencias y revisión del manuscrito fueron realizados por B. Simmons y G. Lamas. A todos ellos mi agradecimiento.

\section{Literatura citada}

Barth, R. 1953. Orgãos odoríferos masculinos de algumas Syntomidae Brasileiras (=Ctenuchidae; Lepidoptera). Mems Inst. Oswaldo Cruz 51(3): 227-262.

Barth, R. 1956. Os tubos abdominais dos machos de alguns Arctiidae e Ctenuchidae do Brasil. Mems Inst. Instituto Oswaldo Cruz 54 (3): 499-515.

Birch, M.C. 1972. Male abdominal brush-organs in British Noctuid Moths and their value as a taxonomic character. Entomologist 105 (1310): 185-205, (1312): 233-244.

Birch, M.C., G.M. Poppy \& T.C. Baker. 1990. Scent and eversible scent structures of male moths. Annu. Rev Entomol. 35: 25-58.

Boppré, M. 1990. Lepidoptera and pyrrolizidine alkaloides.Exemplification of Complexity in Chemical Ecology. J. chem. Ecol. 16: 165-185.
Boppré, M. \& D. Schneider. 1989. The biology of Creatonotus (Lepidoptera: Arctiidae) with special reference to the androconial system. Zool. J. Linn. Soc. 96: 339-356, 10 figs.

Clearwater, J.R. 1975. Structures, development and evolution of the male pheromone system in some Noctuidae. J. Morph. 146: 129-176.

Conner, W.E., T. Eisner, R.K. Vander Meer, A. Guerrero \& J. Meinwald. 1981. Role of precopulatory sexual interaction in an arctiid moth (Utetheisa ornatrix): Role of a pheromone derived from dietary alkaloids. Behav. Ecol. Sociobiol. 9: 227-235.

Davenport, J.W. \& W.E. Conner. 2003. Diertary alkaloids and the development of androconial organs in Estigmene acrea. J. Ins. Sci. 3: 1-6.

Draudt, M. 1915-1917. 3. Family Syntomidae, pp. 33230. In. Seitz, A. (ed.): The Macrolepidoptera of the World. Sttutgart, A. Kernen. 6.

Eltringham, H. 1925. On the abdominal brushes in certain male noctuid moths. Trans. ent. Soc. Lond. 1925: $1-5$.

Hampson, G. 1898. Catalogue of the Lepidoptera Phalaenae in the British Museum. London, British Museum (Natural History). 1:XXII+ 559 pp, 17 pls.

Häuser, C. \& M. Boppré. 1997. A revision of the Afrotropical taxa of the genus Amerila Walker (Lepidoptera: Arctiidae). Syst. Ent. 22: 1-44.

Padgen, H.T. 1957. The presence of coremata in Creatonotus gangis (L.) (Lepidoptera: Arctiidae). Proc. R. ent. Soc. Lond. 32: 90-95.

Scoble, M. 1992. The Lepidoptera: Form, Fuction and Diversity. Oxford. Oxford Unicersity Press.

Speidel, W., H. Fänger \& M. Naumann. 1996. The phylogeny of the Noctuidae (Lepidoptera). Syst. Ent. 21: 219-251.

Travassos, L. 1962. Género Theages Walker, 1885 (Lepidoptera). Nota preliminar. Atas Soc. Biol. Rio de Janeiro 6: 58-59.

Walker, F. 1855. List of the specimens of Lepidopterous Insects in the collection of the British Museum, London 3: 582-775.

Weller, S.J., R.B. Simmons, R. Boada \& W.E. Conner. 2000. Abdominal modification ocurring in wasp mimics of the ctenuchinae-euchromiine clade (Lepidoptera: Arctiidae). Ann. ent. Soc. Am. 93(4): 920-928.

Zerny, W. 1912. Syntomidae.Lep. Catal. 7:179 pp. 